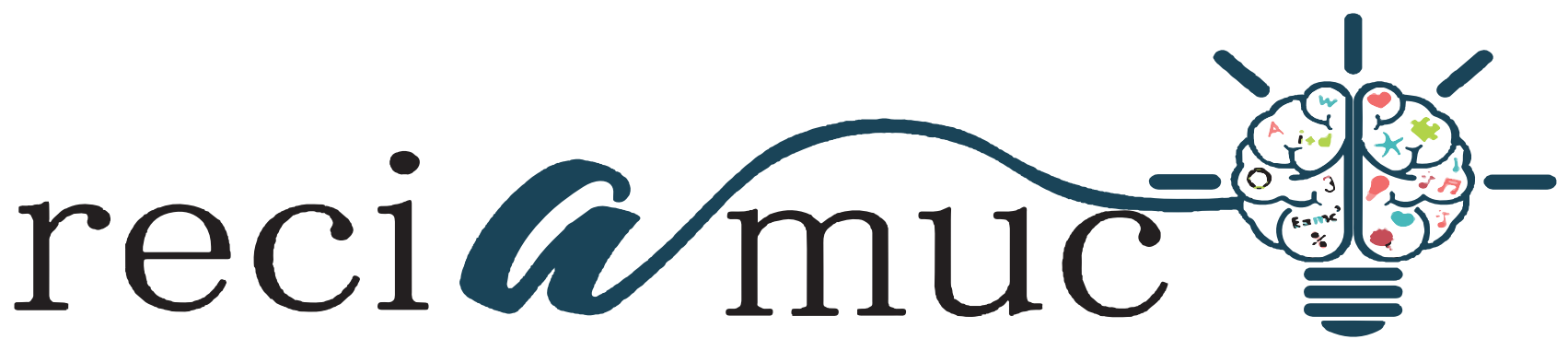

DOI: $10.26820 /$ reciamuc/5.(3).agosto.2021.88-95

URL: https://reciamuc.com/index.php/RECIAMUC/article/view/695

EDITORIAL: Saberes del Conocimiento

REVISTA: RECIAMUC

ISSN: 2588-0748

TIPO DE INVESTIGACIÓN: Artículo de Revisión

CóDIGO UNESCO: 32 Ciencias Médicas

PAGINAS: 88-95

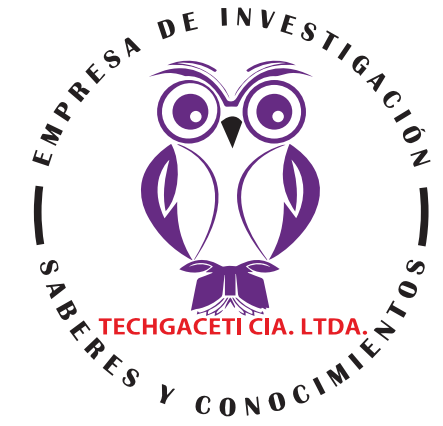

\title{
Complicaciones asociadas a colecistectomía laparoscópica de emergencia
}

Complications associated with emergency laparoscopic cholecystectomy Complicações associadas à colecistectomia laparoscópiça de emergência

Christian Medardo Medina Jiménez'; Silvio Atahualpa Medina Rojas; Silvia Alexandra Medina Jiménez; Jessica Jannette Sotomayor Elizalde ${ }^{4}$

RECIBIDO: 10/06/2021 ACEPTADO: 12/07/2021 PUBLICADO: 31/08/2021

1. Magister en Gerencia en Servicios de la Salud; Médico; Investigador Independiente; Guayaquil, Ecuador; samedina2004@hotmail.com; (D) https://orcid.org/0000-0002-5593-4316

2. Magister en Gerencia en Servicios de la Salud; Doctor en Medicina y Cirugía; Investigador Independiente; Guayaquil, Ecuador; atahualpamedina@hotmail.com; (iD https://orcid.org/0000-0001-8863-0848

3. Medica Especialista en Frenología y Linfologia; Médico; Investigador Independiente; Guayaquil, Ecuador; ysik_sotomayore@hotmail.es; (iD https://orcid.org/0000-0002-6051-173X

4. Magister en Gerencia en Servicios de la Salud; Médico; Investigador Independiente; Guayaquil, Ecuador; silvimedj@gmail.com; (D) https://orcid.org/0000-0003-0329-0368

CORRESPONDENCIA

Christian Medardo Medina Jiménez

samedina2004@hotmail.com

Guayaquil, Ecuador

(c) RECIAMUC; Editorial Saberes del Conocimiento, 2021 


\section{RESUMEN}

La colecistitis aguda es sin duda una de las patologías más comunes en el mundo; se considera la segunda causa de abdomen agudo después de la apendicitis. Es frecuente motivo de consulta a diario en los servicios de urgencias. El presente artículo describe y compara diferentes literaturas acerca de Complicaciones asociadas a colecistectomía laparoscópica de emergencia. Para ello, se recolecta esta información de diferentes fuentes bibliográficas adquiridas de bases de datos (SCOPUS, PubMed, Biblioteca Cochrane, Google Scholar) valorando la calidad y veracidad de la información recopilada, así como la actualidad del contenido. La edad, el estado nutricional, tipo de cirugía y las comorbilidades de los pacientes, son la ecuación perfecta para la aparición de complicaciones asociadas a los procesos quirúrgicos por colecistectomía. El procedimiento por medio de laparoscopia se ha vuelto mucho mas frecuente en la población adulta y sus resultados en la mayoría de los casos son muy eficaces con bajas tasas de complicaciones, ya que es una técnica menos invasiva. Es importante que el diagnostico se realice lo mas temprano posible, ya que la colecistectomía laparoscopia en estos casos produce estancias hospitalarias mas cortas, reduce costos y el tiempo de recuperación es mas acelerado. Hay estudios que afirman y concluyen que la obesidad, cirugías abdominales previas y una edad mayor a los 70 años aumentan considerablemente las probabilidades de complicaciones, así como el sexo femenino representa el mayor porcentaje de complicaciones, por sufrir mas las mujeres de patologías biliares.

Palabras clave: Colecistectomía, laparoscopia, complicaciones, edad, sexo.

\section{ABSTRACT}

Acute cholecystitis is undoubtedly one of the most common pathologies in the world; it is considered the second cause of acute abdomen after appendicitis. It is a frequent reason for daily consultation in the emergency services. This article describes and compares different literatures on Complications associated with emergency laparoscopic cholecystectomy. To do this, this information is collected from different bibliographic sources acquired from databases (SCOPUS, PubMed, Cochrane Library, Google Scholar), assessing the quality and veracity of the information collected, as well as the timeliness of the content. Age, nutritional status, type of surgery and patient comorbidities are the perfect equation for the appearance of complications associated with cholecystectomy surgical processes. The laparoscopic procedure has become much more frequent in the adult population and its results in most cases are very effective with low rates of complications, since it is a less invasive technique. It is important that the diagnosis is made as early as possible, since laparoscopic cholecystectomy in these cases produces shorter hospital stays, reduces costs and the recovery time is faster. There are studies that affirm and conclude that obesity, previous abdominal surgeries and an age greater than 70 years considerably increase the probabilities of complications, as well as the female sex represents the highest percentage of complications, as women suffer more from biliary pathologies.

Keywords: Cholecystectomy, laparoscopy, complications, age, sex.

\section{RESUMO}

A colecistite aguda é, sem dúvida, uma das patologias mais comuns no mundo; é considerada a segunda causa de abdome agudo após a apendicite. É motivo frequente de consultas diárias nos serviços de urgência. Este artigo descreve e compara diferentes literaturas sobre complicações associadas à colecistectomia laparoscópica de emergência. Para isso, essas informações são coletadas de diferentes fontes bibliográficas adquiridas em bancos de dados (SCOPUS, PubMed, Cochrane Library, Google Scholar), avaliando a qualidade e veracidade das informações coletadas, bem como a atualidade do conteúdo. Idade, estado nutricional, tipo de cirurgia e comorbidades do paciente são a equação perfeita para o aparecimento de complicações associadas aos processos cirúrgicos de colecistectomia. O procedimento laparoscópico tem se tornado muito mais frequente na população adulta e seus resultados na maioria dos casos são muito eficazes com baixo índice de complicações, por ser uma técnica menos invasiva. É importante que o diagnóstico seja feito o mais precocemente possível, pois a colecistectomia laparoscópica nesses casos produz menor tempo de internação, reduz custos e o tempo de recuperação é mais rápido. Há estudos que afirmam e concluem que a obesidade, as cirurgias abdominais prévias e a idade superior a 70 anos aumentam consideravelmente as probabilidades de complicações, assim como o sexo feminino representa o maior percentual de complicações, pois as mulheres sofrem mais com patologias biliares.

Palavras-chave: Colecistectomia, laparoscopia, complicações, idade, sexo. 


\section{Introducción}

La colecistitis aguda es sin duda una de las patologías más comunes en el mundo; se considera la segunda causa de abdomen agudo después de la apendicitis. Es frecuente motivo de consulta a diario en los servicios de urgencias (Tapullima Pérez, 2016, pág. 1).

- - La colecistitis crónica es inflamación e irritación de la vesícula biliar que persiste con el tiempo, la colecistitis crónica puede ser el resultado de ataques repetidos de colecistitis aguda (Delgado Cabrera, 2019, pág. 32).

Esta es la clasificación de colecistitis aguda, según la guía de Tokio 2013:

1. COLECISTITIS EDEMATOSA (2 a 4 días): La vesícula tiene fluido intersticial acompañada de dilatación capilar y linfática; la pared se encuentra edematizada.

2. COLECISTITIS NECROTIZANTE (3 a 5 días): la vesícula edematosa presenta cambios con áreas hemorrágicas y necróticas. Cuando la pared vesicular está expuesta a altas presiones, existe obstrucción en el flujo vascular con evidencia histológica de obstrucción y trombosis vascular, aun así no compromete todas las capas tisulares de la pared vesicular.

3. COLECISTITIS SUPURATIVAS ( 7 a 10 días): La pared de la vesícula biliar presenta áreas necróticas y supuradas. En esta etapa la inflamación es evidente, la totalidad de la vesícula comienza a contraerse y la pared se torna más delgada y con proliferación fibrótica .Presenta además abscesos intramurales, sin embargo, no envuelve la totalidad de las capas tisulares de la pared vesicular. Se pueden encontrar abscesos pericolecísticos.

4. COLECISTITIS CRÓNICA. : Esta ocurre cuando hay múltiples episodios inflamatorios, y se caracteriza por atrofia de la mucosa y fibrosis de la pared vesicular. También puede ser causada por una irritación crónica por la presencia de grandes litos que eventualmente pueden ocasionar colecistitis aguda (Tapullima Pérez, 2016, págs. 7-8).

\section{Causas}

La colecistitis aguda litiásica es causada por la obstrucción del conducto cístico, lo que lleva a la distensión de la vesícula biliar, que al distenderse, la circulación sanguínea y el drenaje linfático se ven comprometidos, llevando a la isquemia de la mucosa y generando necrosis (Bocanegra Del Castillo \& Córdova Cuadros, 2013, pág. 114). La causa de colecistitis aguda en su mayoría es la presencia de litos en la vesícula entre un 90-95\% de los casos reportados se deben a esta causa, el porcentaje restante 3,7\% al $14 \%$ son las otras patologías alitiásicas (Collantes Goicochea, 2018).

Las principales causas de colecistitis aguda son:

- Litiásica

- No Litiásica

- Infecciosa:

- En el curso de una sepsis generalizada

- En la fiebre tifoidea

- Debido a gangrena por gérmenes anaerobios

- Química

- Mecánica

- Tumoral

- Vascular

- Parasitaria (Collantes Goicochea, 2018).

\section{Manifestaciones clínicas}

Los pacientes con colecistitis aguda presentan dolor abdominal, siendo común en 
el cuadrante superior derecho o epigastrio. Característicamente, el dolor agudo de colecistitis es constante y severo y generalmente se prolonga (más de cuatro a seis horas). Este dolor puede irradiarse al hombro derecho o la espalda. Los signos y síntomas asociadas pueden incluir fiebre, náuseas, vómitos y anorexia. Es oportuno recolectar antecedentes de ingesta de alimentos grasos una hora o más antes del inicio inicial del dolor. Los pacientes con complicaciones pueden presentar signos de sepsis (gangrena), peritonitis generalizada (perforación), crepitación abdominal (colecistitis enfisematosa) u obstrucción intestinal (íleo biliar) (Ramos Vilchez, 2020, pág. 21).

\section{Diagnóstico}

\section{Laboratorio}

Además de un recuento sanguíneo completo, evaluamos los niveles de lipasa y amilasa sérica, electrolitos, alanina aminotransferasa, aspartato aminotransferasa, bilirrubina, calcio y albúmina para descartar otras causas de dolor abdominal agudo o complicaciones de colecistitis aguda. Se debe realizar una prueba de embarazo en todas las mujeres en edad fértil.

\section{Por imagen}

Ultrasonografía: La presencia de cálculos en la vesícula biliar en el contexto clínico del dolor abdominal en el cuadrante superior derecho y la fiebre respalda el diagnóstico de colecistitis aguda, pero no es diagnóstico. Las características ecográficas adicionales incluyen:

- Engrosamiento de la pared de la vesícula biliar (mayor de 4 a 5 mm) o edema.

- Un "signo de Murphy ecográfico" es similar al signo de Murphy provocado durante la palpación abdominal, excepto que la respuesta positiva se observa durante la palpación con el transductor de ultrasonido. Esto es más preciso que la palpación de la mano porque puede confirmar que efectivamente es la vesí- cula la que está siendo presionada por el transductor de imágenes cuando el paciente recupera el aliento (Ramos Vilchez, 2020, págs. 21-22).

\section{Epidemiología}

- Constituye una de las patologías más frecuentes por las que acude un paciente al servicio de emergencia, y representa aproximadamente de un 20 al 25\% de aquellas patologías que requerirán tratamiento quirúrgico emergente. En informes de autopsias, los cálculos biliares se presentan desde un 11 hasta un 36\%.

- Va a tener un predominio por el sexo, siendo ésta más frecuente en las mujeres en una proporción de 3:2 con respecto a los hombres. La edad más afecta es la que corresponde a la cuarta y sexta década.

- En el Ecuador se considera a la colelitiasis como la segunda causa de morbilidad, precedido por la neumonía; con una tasa de 22,13\% por cada 10000 habitantes. Lo que indica que en una población estimada en el 2013 por el INEC, de 15774749 habitantes, 34.906 de ellos tuvieron egresos hospitalarios por ésta causa (Gavilanes Ibarra \& Quezada Rivera, 2017, págs. 13-14).

\section{Factores de riesgo}

Dentro de los factores de riesgo en general de la colelitiasis, destaca la edad, así en mujeres jóvenes se afecta en $8 \%$, aumentando a 25 a 30\% en mujer adulta; en los varones a mayor edad mayor riesgo. La obesidad, en mujeres es otro factor, un índice de masa corporal mayor de $32 \mathrm{~kg} / \mathrm{m} 2$, incrementa esta posibilidad. Así también la multiparidad, contribuye a colelitiasis. Otro factor a mencionar es perder peso rápidamente, contribuye a la formación de cálculos biliares. En el adulto mayor disminuye esa diferencia entre varón y mujer, a razón de $2 / 1$. Otro factor de riesgo importante son las intervenciones quirúrgicas, siendo más 
complicada cuando se realizó después de las 72 horas (Pisfil Zarpán, 2019, pág. 2).

La colecistectomía laparoscópica en colecistitis aguda constituye un mayor desafío quirúrgico que en la colelitiasis no complicada, porque se presentan mayores dificultades anatómicas, especialmente cuando ya se ha constituido un plastrón vesicular, creándose el riesgo de complicaciones intraoperatorias, de iatrogenia, porcentaje de conversiones y prolongando el tiempo operatorio (Venturelli, Avendaño, , Butte, \& Zamorano, 2018, pág. 9).

\section{Técnica quirúrgica}

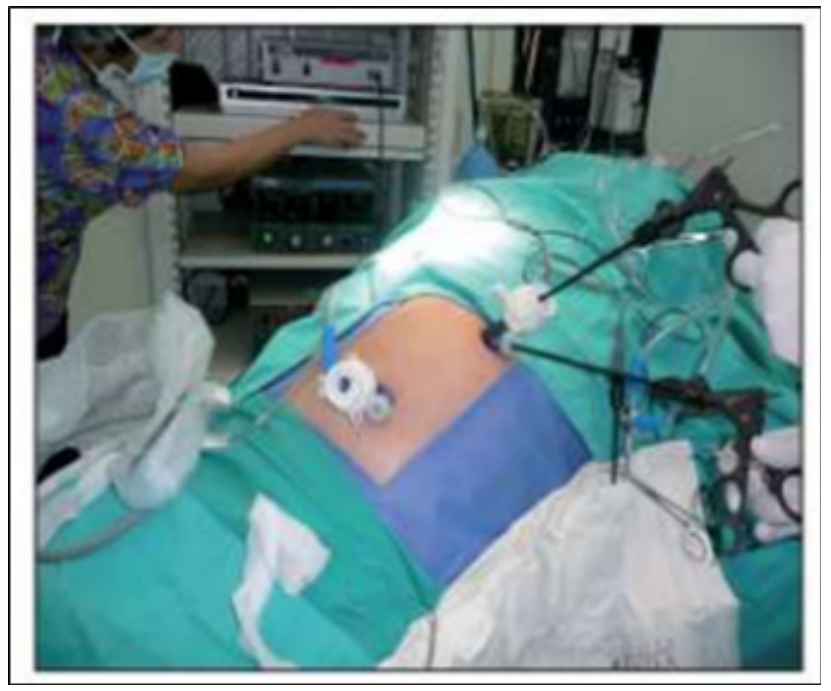

Figura 1. Posición de trocares con dos incisiones de entrada

Fuente: (Bellido Huertas, 2016)

1. Posteriormente se llevará a cabo la realización de neumoperitoneo de acuerdo a técnica habitual con aguja de Veress por mini-incisión en zona umbilical. Colocar el trocar de $10 \mathrm{~mm}$ para la cámara, en la zona umbilical. Luego de la exploración y evaluación de la factibilidad de procedimiento, se hará la incisión epigástrica y la colocación de trocar de 10 mm bajo visión, e inmediatamente proximal a éste, por la misma incisión, se colocará el trocar de $5 \mathrm{~mm}$ que saldrá, sin embargo, en sitio diferente al anterior trocar, pero adyacente al mismo, dejando así un puente de fascia entre ambos. Por este trocar de $5 \mathrm{~mm}$ se levantará y movilizará el polo vesicular.

2. Por último, se realizará el cambio transitorio de la cámara al trocar de $10 \mathrm{~mm}$ en el epigastrio, para colocar bajo visión el trocar de $5 \mathrm{~mm}$ en la zona umbilical, por la misma incisión, pero proximal al trocar de $10 \mathrm{~mm}$ puesto en esta zona, y que saldrá al igual que con los trocares de epigastrio, por un sitio diferente a trocar de $10 \mathrm{~mm}$. Por este trocar de $5 \mathrm{~mm}$ se moviliza y tracciona principalmente el bacinete de la vesícula.

3. La disección se realiza de forma similar a la técnica clásica laparoscópica, con electrocoagulación instrumental por trocar epigástrico, en este caso el distal, que será el canal de trabajo para la introducción de instrumentos para disección, colocación de clips hemostáticos, sección de elementos, tal y cual se hace en la técnica laparoscópica convencional.

4. Una vez liberada la vesícula, se realiza la extracción de la misma por incisión epigástrica o umbilical, con o sin ampliación de aponeurosis según características de la misma. Por último, se procede al cierre de rutina de aponeurosis en ambas incisiones con Vicryl 0 (Bellido Huertas, 2016, págs. 20-21).

\section{Metodología}

El presente artículo describe y compara diferentes literaturas acerca de Complicaciones asociadas a colecistectomía laparoscópica de emergencia. Para ello, se recolecta esta información de diferentes fuentes bibliográficas adquiridas de bases de datos (SCOPUS, PubMed, Biblioteca Cochrane, Google Scholar) valorando la calidad y veracidad de la información recopilada, así como la actualidad del contenido. 


\section{Resultados}

\section{Tabla 1. Complicaciones de colecistectomía}

\begin{tabular}{|c|c|}
\hline \multirow[t]{5}{*}{$\begin{array}{c}\text { Postoperatorias } \\
\text { inmediatas }\end{array}$} & $\begin{array}{l}\text { Hemorragia postoperatoria: Esta se produce tras una colecistectomía } \\
\text { laboriosa o en pacientes con alteraciones de la coagulación, siendo los } \\
\text { cirróticos los pacientes con mayor riesgo. }\end{array}$ \\
\hline & $\begin{array}{l}\text { Absceso subhepático y absceso subfrénico: Esta circunstancia se } \\
\text { produce hasta en el } 5 \% \text { de los pacientes sometidos a cirugía biliar. Dichas } \\
\text { colecciones se infectan, produciendo fiebre, leucocitosis y síntomas } \\
\text { locales en forma de molestias en el hipocondrio derecho. El exudado } \\
\text { biliar de dichas colecciones proviene generalmente de pequeños } \\
\text { canalículos del lecho vesicular, o del propio conducto cístico debido al } \\
\text { deslizamiento de la ligadura. }\end{array}$ \\
\hline & $\begin{array}{l}\text { Peritonitis biliar y fístula biliar: La dehiscencia del muñón cístico es } \\
\text { una complicación relacionada a diversos factores entre los cuales se } \\
\text { encuentra la mala calidad del tejido residual en el nivel de grapado } \\
\text { cístico, debido a inflamación crónica que incluso puede ocasionar } \\
\text { avulsión cística durante el mismo procedimiento si las maniobras de } \\
\text { movilización y tracción no se realizan de manera cuidadosa. El paciente } \\
\text { presenta a las pocas horas de la intervención molestias abdominales sin } \\
\text { focalidad, con sensación de náuseas, febrícula y malestar general. }\end{array}$ \\
\hline & $\begin{array}{l}\text { Fístula colecistoduodenal: Aunque se trata de un tejido sano y bien } \\
\text { vascularizado, la fístula duodenal puede producirse siendo una } \\
\text { complicación grave que suele requerir una reintervención precoz con el } \\
\text { riesgo de sepsis grave. }\end{array}$ \\
\hline & $\begin{array}{l}\text { Coledocolitiasis y pancreatitis: Aunque hoy día la tasa global de } \\
\text { coledocolitiasis durante una colecistectomía ha disminuido por debajo del } \\
5 \% \text {, debido en gran parte a la precocidad del tratamiento de la colelitiasis, } \\
\text { hasta en un } 30 \% \text { es responsable de las reintervenciones biliares, o de los } \\
\text { abordajes endoscópicos postoperatorios. El diagnóstico suele sospecharse } \\
\text { al realizar la colangiografía transKehr. }\end{array}$ \\
\hline $\begin{array}{c}\text { Postoperatorias } \\
\text { tardías }\end{array}$ & $\begin{array}{l}\text { Ictericia, colestasis y estenosis biliar: Existen otro tipo de lesiones } \\
\text { biliares en relación con ligaduras, contusiones, quemaduras con el bisturí } \\
\text { eléctrico, elongaciones de la vía por excesiva tracción o lesiones } \\
\text { isquémicas, que se reducen tras intervenciones de cirugía biliar, de } \\
\text { trasplante hepático o incluso tras traumatismos. } \\
\text { En estos pacientes la evolución hacia un cuadro de colestasis es la regla } \\
\text { produciendo las complicaciones locales y generales de dicha situación. La } \\
\text { lesión que aparece a medio plazo es una estenosis benigna de las vías } \\
\text { biliares. En esta situación, el signo clínico más relevante es la colangitis } \\
\text { seguida de ictericia, que aparecerá en la mayoría de los casos en las } \\
\text { siguientes semanas o meses de la operación }\end{array}$ \\
\hline
\end{tabular}

Fuente: (Venegas Quenta, 2020). Elaboración Propia. 


\section{Otras complicaciones}

1. Barotrauma: Esta se puede presentar por hiperinsuflación, con el uso de insufladores no automáticos o que no tengan medidores de presión intraabdominal; pero aun con medidores existe la posibilidad de que dichas válvulas se obstruyan. Este puede ser evidente al observar que no hay desplazamiento del diafragma, y las máquinas de anestesia marcan presiones de insuflación elevadas; al no detectarse la primera manifestación puede ser la presencia de arritmias cardíacas que pueden ser mortales, puede encontrarse como manifestación tardía la absorción de C02 y trastornos en el equilibrio ácido básico.

2. Insuflación Extraperitoneal: Es una complicación menor que puede ser de gran magnitud, incomodar e incluso impedir la cirugía de no detectarse. Se evita con la correcta colocación de la aguja de Veress en la cavidad abdominal y estando siempre atentos a la presión intraabdominal al inicio de la insuflación, ya que al aumentar rápidamente se deberá recolocar la aguja.

3. Enfisema Subcutáneo: El enfisema subcutáneo ocurre por lo general cuando no penetra la punta de la aguja de Veress en la cavidad peritoneal antes de insuflar el gas. Esto puede ocasionar que se acumule este último en el tejido subcutáneo o entre la fascia y el peritoneo.

4. Embolia gaseosa: Puede ocurrir al inicio de la operación, cuando se canaliza un vaso del epiplón, también cuando se tiene un lecho hepático sangrante y problemático con un seno venoso abierto y existe hiperinsuflación. Su tratamiento consiste en evacuar el neumoperitoneo, colocar al paciente en posición de Durant y extracción del C02 por medio de un catéter central en la aurícula derecha o en el ventrículo.

5. Lesiones con la aguja de Veress o con trocares: Estas suelen suceder durante la primera punción, ya que es el único procedimiento ciego para abordar la cavidad abdominal, se debe tener cuidado con la penetración de los instrumentos en caso de cirugías previas, ya que la posibilidad de vísceras adheridas a la pared, puede ocasionar lesión de las mismas.

6. Hemorragia: Esta puede ocurrir en cualquier momento de la cirugía, desde la insuflación hasta el retiro del último trocar. El sangrado producido por la aguja de Veress, por lo general se controla por si solo o por la acción de agentes vasopresores, y al lesionarse vasos del epiplón es preferible ligar o coagular. Otro tipo de sangrado puede ser ocasionado por desgarramiento o mala ligadura de la arteria cística o una de sus ramas, como también por lesión del hígado por excesiva tracción vesicular o agresión instrumenta (Domínguez Membreño, 2016, págs. 19-21).

\section{Conclusiones}

La edad, el estado nutricional, tipo de cirugía y las comorbilidades de los pacientes, son la ecuación perfecta para la aparición de complicaciones asociadas a los procesos quirúrgicos por colecistectomía. El procedimiento por medio de laparoscopia se ha vuelto mucho mas frecuente en la población adulta y sus resultados en la mayoría de los casos son muy eficaces con bajas tasas de complicaciones, ya que es una técnica menos invasiva. Es importante que el diagnostico se realice lo mas temprano posible, ya que la colecistectomía laparoscopia en estos casos produce estancias hospitalarias mas cortas, reduce costos y el tiempo de recuperación es mas acelerado. Hay estudios que afirman y concluyen que la obesidad, cirugías abdominales previas y una edad mayor a los 70 años aumentan considerablemente las probabilidades de complicaciones, así como el sexo femenino representa el mayor porcentaje de compli- 
caciones, por sufrir mas las mujeres de patologías biliares.

En vista de las múltiples complicaciones que se pueden presentar en la colecistectomía, es importante que el abordaje medico, sea con personal altamente capacitado y con experiencia, así como la correcta realización de las historias clínicas para el estudio de las comorbilidades que pueda tener cada paciente.

\section{Bibliografía}

Bellido Huertas, J. (2016). Factores clínico-epidemiológicos asociados a complicaciones post colecistectomía laparoscópica en adultos mayores atendidos en el Hospital José Agurto Tello de Chosica 2013-2015.

Bocanegra Del Castillo, R. R., \& Córdova Cuadros, M. (2013). Colecistectomía laparoscópica en el adulto mayor: complicaciones postoperatorias en mayores de 75 años en el Hospital Nacional Cayetano Heredia, Lima, Perú, del 2007-2011. Revista de Gastroenterología del Perú, 33(2), 113-120.

Collantes Goicochea, J. B. (2018). Análisis comparativo de las Complicaciones de la colecistectomía convencional versus colecistectomía laparoscópica en cirugía de emergencia en el Hospital Nacional Sergio E. Bernales en los meses de EneroSeptiembre del 2017.

Delgado Cabrera, J. (2019). Factores de riesgo asociados a conversión de colecistectomía laparoscópica a colecistectomía convencional en el servicio de cirugía del Hospital Nacional Dos de Mayo durante el periodo Enero-Noviembre 2018.
Domínguez Membreño, J. D. (2016). Incidencia de complicaciones asociadas a colecistectomía laparoscópica en pacientes intervenidos quirúrgicamente en el Hospital Escuela Antonio Lenin Fonseca, durante el 2015.

Gavilanes Ibarra, Á. C., \& Quezada Rivera, C. (2017). Estudio comparativo de colecistectomía abierta vs colecistectomía laparoscópica en pacientes que han sido operados en emergencia del Hospital Teodoro Maldonado Carbo en el periodo octubre del 2016 a enero del 2017.

Pisfil Zarpán, C. M. (2019). Morbimortalidad por colecistectomía laparoscópica en colecistitis aguda en el adulto mayor en el Servicio de Emergencia del Hospital de Huaral año 2015-2018.

Ramos Vilchez, L. A. (2020). Factores asociados a complicaciones post Colecistectomía laparoscópica en el Hospital San José del Callao, 2018.

Tapullima Pérez, M. J. (2016). Características Clínico Epidemiológicas y Quirúrgicas de colecistectomías laparoscópicas convertidas en el Servicio de Emergencia; en pacientes con colecistitis aguda en el Hospital Nacional Daniel Alcides Carrión desde 2013 al 2015.

Venegas Quenta, J. A. (2020). Factores asociados a complicaciones post colecistectomía laparoscópica en pacientes con patología biliar en el Servicio de cirugía general del Hospital Hipólito Unanue de Tacna, enero a diciembre del 2019.

Venturelli, A., Avendaño, , R., Butte, J., \& Zamorano, I. (2018). Colecistectomía laparoscópica en colecistitis aguda. Cuadernos de Cirugía, 14(1), 9-11.

\section{CITAR ESTE ARTICULO:}

Lara Icaza, J. D., Triana Castro, C. T., \& Fuenmayor Boscán, A. (2021). Helicobacter pylori y los diferentes métodos para el diagnóstico: invasivos y no invasivos. RECIAMUC, 5(3), 73-87. https://doi.org/10.26820/reciamuc/5.(3). agosto.2021.73-87

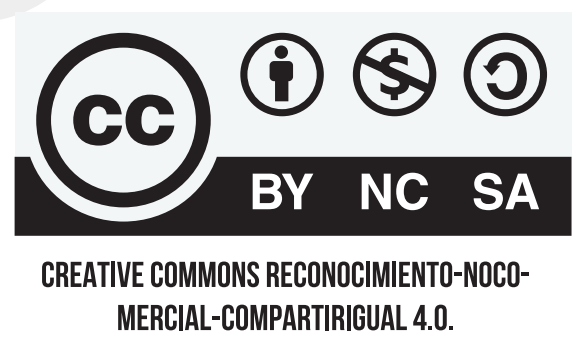

